Instituto Internacional de Investigación y Desarrollo Tecnológico Educativo INDTEC, C.A.

DOI: https://doi.org/10.29394/Scientific.issn.2542-2987.2020.5.16.6.120-140

OAI-PMH: http://www.indteca.com/ojs/index.php/Revista Scientific/oai

Artículo Original / Original Article

\title{
Acercamiento a una Experiencia Formativa de Teatro Comunitario
}

Autor: Igor Raphael Martínez Rebolledo

Universidad de los Andes, ULA

igorm@ula.ve

Mérida, Venezuela

https://orcid.org/0000-0002-7853-434X

\section{Resumen}

El artículo expone el proceso formativo y de investigación aplicado en una experiencia de teatro comunitario desarrollada en los años 2017 - 2018, en la comunidad del Chama, Municipio Libertador de la ciudad de Mérida, Venezuela. El Paradigma de la investigación fue cualitativo y se desarrolló bajo el enfoque: Introspectivo vivencial. La metodología aplicada, fue de AcciónParticipación, la cual se desplegó en cuatro fases de trabajo: de escritura dramática, de taller de formación, de puesta en escena y de representación. El proceso se registró a través de la técnica de observación participativa, el cual generó importantes reflexiones en respuesta al objetivo de la investigación el cual se orientó hacia la práctica recreativa, formativa e integradora del Teatro, como una alternativa para el reconocimiento de las potencialidades de jóvenes adolescentes en estado de vulnerabilidad, a favor de una experiencia de intercambio y práctica humana para la inclusión de jóvenes en la comunidad.

Palabras clave: participación comunitaria; teatro; educación comunitaria; animación cultural.

Cómo citar este artículo:

Martínez, I. (2020). Acercamiento a una Experiencia Formativa de Teatro Comunitario. Revista Scientific, 5(16), 120-140, e-ISSN: 2542-2987. Recuperado de: https://doi.org/10.29394/Scientific.issn.2542-2987.2020.5.16.6.120-140

Fecha de Recepción:

09-12-2019
Fecha de Aceptación:

03-03-2020
Fecha de Publicación:

05-05-2020 
OAI-PMH: http://www.indteca.com/ojs/index.php/Revista_Scientific/oai

Artículo Original / Original Article

\title{
Approach to a Community Theater Training Experience
}

\begin{abstract}
The article exposes the training and research process applied in a community theater experience developed in the years 2017 - 2018, in the community of Chama, Libertador Municipality of the city of Mérida, Venezuela. The research paradigm was qualitative and developed under the approach: Experiential introspective. The methodology applied was Action-Participation, which was deployed in four phases of work: dramatic writing, training workshop, staging and representation. The process was recorded through the participatory observation technique, which generated important reflections in response to the objective of the research which was oriented towards the recreational, formative and integrative practice of the Theater, as an alternative for the recognition of the potentialities of young adolescents in a state of vulnerability, in favor of an experience of exchange and human practice for the inclusion of young people in the community.
\end{abstract}

Keywords: community participation; theatre; community education; cultural animation.

How to cite this article:

Martínez, I. (2020). Approach to a Community Theater Training Experience. Revista Scientific, 5(16), 120-140, e-ISSN: 2542-2987. Recovered from: https://doi.org/10.29394/Scientific.issn.2542$\underline{2987.2020 .5 .16 .6 .120-140}$

Date Received:

09-12-2019
Date Acceptance:

03-03-2020
Date Publication:

05-05-2020 


\section{Introducción}

En Venezuela en los últimos años, el Teatro Educativo se ha convertido en una herramienta pedagógica en el aula de clase en respuesta a la necesidad, no sólo de que nuestros niños, niñas y adolescentes aprendan de manera divertida y creativa, sino que también desarrollen competencias fundamentales como la capacidad para expresarse y comunicarse. Se trata de una experiencia de juego-educativo que, dentro del transcurso formativo integral de nuestros niños, niñas y jóvenes, promueve de manera efectiva la autonomía y el desarrollo de la autoestima, aspectos generadores de placer y conocimiento individual y colectivo, porque participan de una experiencia teatral y aprenden de ella a reconocerse y a conocer a los demás. Estas dinámicas de "juego" insertas en el Teatro educativo, responden a lo planteado por el Fondo de las Naciones Unidas para la Infancia (UNICEF, 2018), cuando expresa acerca del tema que:

[...] Constituye una de las formas más importantes en las que los niños pequeños obtienen conocimientos y competencias esenciales. Por esta razón, las oportunidades de juego y los entornos que favorecen el juego, la exploración y el aprendizaje práctico constituyen el fundamento de los programas de educación preescolar eficaces (pág. 7).

Nos convertimos entonces en sujetos y objetos de conocimiento y comunicación, principalmente porque se trata de una actividad que fortalece el trabajo en equipo y donde se comparten vivencias, fortalecen valores y se incentiva confianza dentro de un desafío creativo. En este sentido, Vargas (2015), sostiene que:

[...] La activación del razonamiento, el mejoramiento actitudinal y el fortalecimiento de la autovaloración, el autoestima y la confianza personal son posibles si se combina de manera adecuada la lúdica en el proceso formativo de cualquier índole, brindando de este modo una infinidad de variadas aplicaciones (pág. 1). 
Es el "Juego" un punto de partida para abordar la representación de la realidad y es una de las estrategias por excelencia utilizadas por los docentes, principalmente de los primeros años de formación primaria, con el propósito de diagnosticar, a partir de acciones y situaciones simuladas, las características sociales del niño, sus posibilidades expresivas y de comunicación y su consideración acerca de su entorno más inmediato y del mundo que le rodea.

Esta manera de entender el Teatro Educativo como una herramienta para hacer del teatro como expresión artística un medio para aprender y contribuir en la formación integral de niños, niñas y adolescentes en formación, ha sido motivo de estudio vinculado a teorías psicológicas y del aprendizaje y a la determinación de prácticas en las que la dramatización se ha convertido en puente para generar aprendizaje en la escuela.

Al respecto, Palmero (2008), en su análisis acerca de la teoría del aprendizaje, menciona que: "[...] se trata de una teoría constructivista, ya que es el propio individuo-organismo el que genera y construye su aprendizaje" (pág. 9). Para ello resulta necesaria la intervención del Teatro educativo a manera de que las colectividades se integren desde de una experiencia formativa teatral como una estrategia para expresar su voz. Es mediante el juego teatral, que el individuo comienza a comprometerse a ser sujeto y objeto de conocimiento, principalmente porque se trata de una actividad que fortalece el trabajo en equipo y donde se comparten vivencias, nuevas formas de comunicación, fortalecimiento de valores y confianza dentro de un desafío creativo. Por su parte, Cruz (2014), expresa que:

La utilización en el aula de actividades dramáticas permiten que los alumnos desarrollen sus capacidades artísticas; puedan tener más confianza para expresar sus sentimientos y emociones porque hará más ágiles y seguros su imaginación, voz y movimientos; obtendrán más herramientas de comunicación y trabajarán de forma más amena objetivos y contenidos del currículum (pág. 75). 
Este planteamiento refuerza la importancia del Teatro como vehículo para expresar la memoria, el sentir y las necesidades de un colectivo, ya que es precisamente este, fundamentado en los métodos técnicos y formativos del Teatro educativo, quien abre la puerta a una mirada responsable a la hora de plantear un mensaje que, si bien plantea problemas que forman parte de una realidad y sus posibles soluciones, también es capaz de convertirse en un canal para pronunciar valores, sentires, ambiciones y una mirada artística de la realidad con la voluntad de integración que debe existir entre los miembros de una colectividad.

Es por eso que esta experiencia resulta fundamental en nuestras comunidades venezolanas, las cuales se han ido convirtiendo en espacios muy diversos y complejos que presentan múltiples problemas y necesidades que deben ser abordados de manera conjunta: familia, escuela y comunidad. A esto se suma la gran preocupación que existe en nuestras comunidades en relación a la cantidad de jóvenes en edad escolar y liceísta que en la actualidad han desertado del sistema educativo y que se encuentran en estado de vulnerabilidad, pues las drogas (consumo y distribución) y la delincuencia, entre otras, ante las necesidades económicas que embarga a sus núcleos familiares, los colocan en una fácil posición para delinquir.

Para ello se hace necesario incorporar al proceso de intervención cultural y participación comunitaria, un conjunto de valores y actitudes que permitan a los diferentes actores de un grupo o colectivo, superar las diferencias y dificultades históricas que se puedan presentar a la hora de llevar a la práctica métodos participativos de manera conjunta, sobre todo, cuando uno de sus principales problemas es la situación difícil en la que se encuentran sus generaciones futuras, de allí que el docente en prácticas sociales de investigación asuma retos orientados a la transformación social a partir de diferentes proyectos educativos en las comunidades. Ejemplo de ello, es lo planteado por Flores, Loaiza y Rojas (2020), cuando mencionan que: 
[...] El docente de la unidad curricular Proyectos asume funciones de investigador, tutor, asesor, facilitador y mediador de aprendizajes, que lo llevan a formar a un individuo con visión crítica, creativa y reflexiva ante las demandas sociales, considerándolo un recurso o talento intelectual para el desarrollo de las capacidades científico-tecnológicos como fuente innovadora de transformación social (pág. 109).

Es esta la razón por lo que se hace importante dentro de nuestras comunidades la corresponsabilidad con el propósito de asumir el carácter activo, formativo y transformador con el cual se debe identificar cada miembro de estos colectivos y la responsabilidad que deberá poseer cada actor partícipe de estos.

En el contexto del estado Mérida, las colectividades están inmersas dentro de una realidad muy particular en lo que se refiere a formas de integración comunitaria, principalmente porque a pesar de los esfuerzos educativos, especialmente los que se han orientado desde el Proyecto Educativo Integral Comunitario (PEIC), el cual constituye un programa bandera del Ministerio del Poder Popular para la Educación y que se ha convertido en plataforma para abrir caminos en la conjunción familia-escuela-comunidad y que según Parra (2009), citado por Capitillo, Urribarri y Materano (2014), se trata de un:

[...] Intercambio de experiencias, de compromisos, de visión, en vinculación del plantel con la comunidad, este, se fundamenta en una concepción holística y específica del hecho educativo, en él se analizan las necesidades y aspiraciones del colectivo que conforma el plantel y su entorno (pág. 4).

A pesar de estos esfuerzos se ha ido abandonando la conciencia ciudadana, gran parte de los merideños ha olvidado la importancia de que la comunidad requiere del concurso de todos sus integrantes para llevar adelante proyectos significativos que repercutan en una mejor condición de vida.

Es ese el caso específico de la comunidad de El Chama, ubicada en el 
Municipio Libertador de la ciudad de Mérida, una colectividad que, aun no escapando a esta realidad, ha ido marcando diferencias al abrir sus puertas, no sólo para reconocer parte de sus debilidades, sino también para enfrentar en colectivo sus dificultades, tales como lo que han venido afrontando en los últimos años con la deserción de jóvenes adolescentes de las aulas de clase, viéndolos vulnerables al ocio y con el riesgo de formar parte de la delincuencia del país.

Fueron estas reflexiones las que motivaron la presente investigación orientada a propiciar un acercamiento a una experiencia formativa de teatro comunitario dirigida a jóvenes adolescentes del mencionado sector con el propósito de desarrollar una práctica de una actividad teatral formativa y de producción que se convirtiera en una alternativa para el reconocimiento de las potencialidades de jóvenes en estado de vulnerabilidad para que se vieran motivados a retornar a las aulas de clases.

Toda una experiencia de teatro comunitario que generó reflexiones en respuesta al objetivo de la investigación planteado, el cual se orientó hacia cómo la práctica recreativa, formativa e integradora del Teatro, puede llegar a convertirse en una alternativa para el reconocimiento de las potencialidades de jóvenes adolescentes en estado de vulnerabilidad, experiencia que estuvo llena de grandes aciertos, pero también de contingencias, donde facilitadores, participantes y la colectividad misma, se involucraron en el saber, conocer y finalmente aprender a partir de una experiencia humana, creativa, de comunicación, de dar y recibir bidireccionalmente y desde donde se aprendió también de lo que estaba y no, previsto en la planificación.

\section{Metodología}

La metodología planteada respondió a la necesidad de encauzarse hacia una visión muy personal del objeto de estudio en el que el paradigma cualitativo se abrió hacia el desarrollo de procesos creativos, formativos y 
cooperativos encausados para desarrollar una experiencia en la que el Teatro comunitario se convirtiera en una plataforma para generar integración e intercambio de saberes en una comunidad popular de la ciudad de Mérida, Venezuela. En este sentido, Strauss y Corbin (2002), en relación a la investigación cualitativa, manifiestan que se trata de:

[...] Cualquier tipo de investigación que produce hallazgos a los que no se llega por medio de procedimientos estadísticos $u$ otros medios de cuantificación, puede tratarse sobre la vida de la gente, las experiencias vividas, los comportamientos, emociones y sentimientos, así como al funcionamiento organizacional, los movimientos sociales, los fenómenos culturales y la interacción entre las naciones (págs. 12).

Partiendo de este paradigma se determina como enfoque investigativo la introspectiva vivencial, orientada, tal y como lo entendemos de Padrón (2001): es una interpretación de simbolismos socioculturales en la que los seres humanos implicados abordan la realidad según las características del grupo social al que pertenecen, estableciendo así un "acto" de comprensión de la realidad en el que los procesos investigativos se convierten en mecanismos de transformación y emancipación humana. La investigación acción participación se desarrolló partiendo de un diagnóstico de la situación y la elaboración de un plan de acción.

La observación participativa como técnica de investigación brindó la oportunidad de elaborar un registro escrito de toda la experiencia, proceso en el que se reconocieron las incidencias e interacciones establecidas a lo largo de las acciones de intervención, mismas que se fueron desarrollando según lo planteado en el plan de acción. Todos los episodios expresados en cada una de las distintas fases de trabajo fueron registrados, codificados e interpretados para dar mayor especificidad a los resultados de la investigación en función de establecer un acercamiento formativo de Teatro comunitario en una comunidad de la ciudad de Mérida, Venezuela. 


\subsection{La propuesta de Investigación}

La experiencia de Teatro comunitario, en la comunidad del Chama, Municipio Libertador de la ciudad de Mérida, Venezuela, nació de la urgente necesidad de generar un trabajo comunitario que permitiera desplegar una experiencia formativa triádica, integrada por los siguientes aspectos: Organización comunitaria, impulso creativo de jóvenes de una comunidad y formación integral de jóvenes adolescentes en situación de vulnerabilidad, a manera de comprobar que en dicho proceso se podía contribuir en la transformación que hiciera posible que la población impactada, en este caso jóvenes y miembros de la comunidad de la Cuenca del Chama, lograrán concebir su entorno como un espacio para la paz, solidaridad e integración comunitaria. Principios estos que le son propios al momento histórico que vivimos en nuestro país.

Para ello fue necesario que el investigador solicitara un derecho de palabra al Consejo Comunal de la Cuenca del Chama en la Parroquia San Jacinto, contexto específico seleccionado para llevar adelante la investigación, debido a que cómo es tradicional, cualquier experiencia de participación comunitaria con estas características, amerita la presentación de un proyecto ante los líderes de la comunidad. Fue allí donde se dio la oportunidad de abrir la puerta a un debate de ideas acerca de la aplicabilidad del proyecto de Teatro comunitario y solicitar el apoyo de los grupos organizados para llevarlo adelante.

Una vez establecidos en la Escuela Mahatma Gandhi, ubicada en la comunidad como sede operativa, se diseñaron planes de trabajo teatral con el propósito de contribuir a proporcionar experiencias pedagógico-recreativas que canalizarán las inquietudes de doce jóvenes adolescentes, todos varones, seleccionados por el consejo comunal bajo el consentimiento de sus representantes, debido a que se encontraban en estado de vulnerabilidad en la Cuenca del Chama y que no encontraban opciones y ocupaciones que 
fueran verdaderamente productivas en el avance de todas sus potencialidades.

\subsection{El diagnóstico}

La experiencia de Teatro comunitario, inició el día 11 de Julio del año 2017, en el que se le propuso a la comunidad del Chama un proyecto que establecía dos fases de intervención: una de diagnóstico de la comunidad y una consecuente de aplicación de la experiencia formativa de Teatro comunitario, propuesta que, una vez aprobada, se pondría en marcha en los espacios del Liceo Luis Beltrán Prieto Figueroa, sector "Cinco Águilas Blancas" Parroquia Jacinto Plaza, de "El Chama”, estado Mérida, Venezuela.

Se llevó adelante un diagnóstico utilizando un instrumento de recolección de información a manera de establecer un marco referencial del contexto, proceso que generó un plan de acción o intervención en correspondencia con las fortalezas y debilidades de la comunidad objeto de estudio. Esta experiencia suministró valiosos insumos para la ejecución de los componentes y líneas de acción contenidas en la programación. En ella, se plantearon una serie de estrategias orientadas al propósito de alcanzar una práctica, en la que doce jóvenes adolescentes del sexo masculino, miembros de la Comunidad del Chama, cuyas edades oscilaban entre 14 y 17 años, fortalecieran vínculos partiendo de una vivencia recreativa y formativa en torno a las artes escénicas.

\subsection{El diseño del plan de acción}

Se plantearon una serie de estrategias orientadas al propósito de que los jóvenes adolescentes fortalecieran vínculos partiendo del Teatro como forma de acción participativa. Así mismo se contempló dentro de las acciones realizar un registro escrito producto de la observación en donde se reflejaría la experiencia expresada en interacciones establecidas en los actos, acciones, 
incidencias y acontecimientos del plan. Se planificaron entonces cuatro fases de trabajo en la que estuvieron propuestas diversas actividades denominadas "acciones", planteadas de manera tal, que el grupo participante pudiera desarrollar una práctica en la que vivenciaran: la escritura dramática, tal y como se evidencia en el fragmento del plan de acción expresado en la tabla 1 , y un taller de formación, toda una experiencia para dar valor al trabajo en equipo en forma cooperativa con el propósito común de elaborar una puesta en escena y representarla en la comunidad.

Tabla 1. Fragmento de plan de acción.

\begin{tabular}{|c|c|}
\hline ACTIVIDAD & $\begin{array}{l}\text { FASE I: Escritura dramática } \\
\text { Acción 1: De orientación acerca de las técnicas y formas } \\
\text { expresivas para abordar la experiencia de la escritura teatral. }\end{array}$ \\
\hline CONTENIDO & $\begin{array}{l}\text { - Aspectos importantes de la obra dramática en el aula. } \\
\text { - Técnica de la escritura teatral. }\end{array}$ \\
\hline ESTRATEGIAS & $\begin{array}{l}\text { - Charla informativa Exposición, demostración y orientación. } \\
\text { - Discusión y debate acerca de temas de orden comunitario. }\end{array}$ \\
\hline RECURSOS & $\begin{array}{l}\text { - Sede del Consejo comunal del Chama; Cartelera; Cuadernos; } \\
\text { Hojas blancas; Lápices. }\end{array}$ \\
\hline FECHA & Del 16 de septiembre al 14 de octubre del año 2017. \\
\hline RESPONSABLES & Investigador / Facilitador del proceso. \\
\hline EVALUACIÓN & $\begin{array}{l}\text { - Instrumento de evaluación. } \\
\text { - Registro en escrito de la experiencia. } \\
\text { - Reflexión acerca de la producción escrita y los testimonios de los } \\
\text { participantes con relación a la experiencia. }\end{array}$ \\
\hline
\end{tabular}

Fuente: El Autor (2017).

\subsection{La ejecución del plan de acción}

En cuanto a la ejecución de las acciones de la programación, las mismas previeron un diseño de plan formativo sujeto a los cambios que pudiesen producirse en el desarrollo de la experiencia. Cada fase debía orientarse a generar un mayor conocimiento acerca de la importancia de una experiencia recreativa, formativa, integradora y social del Teatro comunitario y de sus posibilidades como alternativa para el reconocimiento de las potencialidades de jóvenes adolescentes.

El punto de partida fue determinar lo que para cada uno de los jóvenes 
participantes significaban Los valores como elementos fundamentales para reforzar la conciencia ciudadana estableciendo procesos de reflexión acerca autorreconocimiento de su conducta cívica en correspondencia con la forma de vida de su comunidad. Fue a partir de la disertación, escritura y toda la experiencia creativa orientada en la primera fase de la experiencia que se logró proyectar un texto dramático para una posible representación escénica.

\subsubsection{Fase I: de escritura dramática}

Se desplegó desde el día 16 de septiembre al 14 de octubre del año 2017 , a partir de las 2:30 pm, hasta las 5:30 pm. Se organizó al grupo en tres equipos de trabajo. La fase se estructuró en cuatro acciones, en donde se plantearon ejercicios de escritura para la generación del drama y en donde podían expresar sus ideas en forma oral posibilitando a un compañero de cada equipo transcribir las ideas una vez socializada la información.

En la primera acción se partió de una premisa de la escritura del drama denominada: ¿Qué quiero decir? a partir de la cual surgieron diferentes temas considerados muy relevantes por los jóvenes participantes para el posterior desarrollo de la investigación. Escogieron como temas el embarazo precoz y la importancia del aseo personal en función de una mejor convivencia. La segunda acción, se orientó a la técnica de la escritura y sus posibilidades expresivas, la tercera hacia la producción grupal en la que realizaron un texto en conjunto y la cuarta se dedicó al espacio de la reflexión, en donde se leyeron los textos resaltando en la voz de cada participante la importancia de los temas seleccionados para la escritura.

En el desarrollo de esta fase de trabajo, al tener que enfrentar la escritura en el ejercicio: ¿Qué quiero decir? La primera reacción fue de rechazo, pues algunos de estos jóvenes no dominaban muy bien la lectura ni la expresión escrita de ideas, este choque resultó importante en la medida en que fueron surgiendo propuestas para la realización del texto escrito aún y con 
las debilidades para poder trasladarlas en su totalidad a la escritura.

\subsubsection{Fase II: del Taller de formación}

Realizada entre los días 18 de octubre al 14 de diciembre del año 2017, de 2:30 pm, a 5:30 pm, en los espacios del Liceo Luis Beltrán Prieto Figueroa, sector "Cinco Águilas Blancas" Parroquia Jacinto Plaza, Comunidad de "El Chama".

En este segundo momento se plantearon una serie de acciones con el propósito de desarrollar un plan de ejercicios conducentes a tomar conciencia acerca de lo que significa el "miedo escénico" y el "temor al fracaso" y cómo encausar las intenciones representativas a favor de la expresión y comunicación. La fase propuso establecer una serie de tareas a partir de juegos teatrales donde los participantes se desinhibieran, tuvieran la oportunidad de conocerse, reconocer sus capacidades expresivas y de comunicación y participar en colectivo en una actividad muy diferente.

\subsubsection{Fase III: de la Puesta en escena}

La tercera fase de trabajo del Plan de Acción, comenzó el lunes 25 de abril y culminó el miércoles 27 de junio del año 2018. Se desarrolló en jornadas de trabajo de 2:30 pm a 5:00 pm en el Liceo Luis Beltrán Prieto Figueroa, sector "Cinco Águilas Blancas" Parroquia Jacinto Plaza, Comunidad de "El Chama" Se inició con la lectura y puesta en escena de los textos teatrales ensamblados a partir de las producciones escritas de cada uno de los participantes. Dichos textos de creación colectiva recibieron los nombres de "La historia de Juan el Sucio" y "Llevas una estrella en tu vientre". En ambas se exponen reflexiones acerca de la importancia de los valores humanos ante estas problemáticas comunitarias.

Para ello se incorporaron padres y representantes, así como también miembros de la comunidad a manera de contribuir en los diseños y realización 
de vestuario y escenografía. Fue una jornada muy productiva y creativa de ensayos. Llegado este momento los jóvenes estaban integrados en una dinámica de trabajo que facilitó mucho la producción colectiva. Algunos padres y representantes tuvieron la oportunidad de asistir a los ensayos y presenciar de qué se trataba esta actividad en la que estaban involucrados sus representados.

\subsubsection{Fase IV: de La representación}

Esta fase se desarrolló el día 30 de junio del año 2018 en horario de 5:00 pm a 8:00 pm.

Acción 1: Para llevar adelante esta acción se requirió el apoyo de un especialista en maquillaje teatral, quien dictó un taller sobre la realización de maquillajes característicos. Esta experiencia contribuyó a que los jóvenes participantes se familiarizaran con las técnicas básicas de maquillaje y propusieran sus propios diseños para los personajes que debían representar. El taller tuvo una fase expositiva acerca de la importancia del maquillaje en la obra teatral y posteriormente se presentaron imágenes de los diferentes caracteres que se obtienen del maquillaje. Finalmente se explicó la técnica de aplicación y cada participante tuvo la oportunidad de maquillar a un compañero según cada personaje.

Acción 2: Para llevar adelante esta acción se requirió del apoyo de algunos miembros de la comunidad y del mismo Consejo Comunal, quienes contribuyeron a montar los elementos escenográficos para la representación en la Cancha de la Comunidad del Chama, colocar un pequeño sistema de iluminación para apreciar la obra en un espacio no convencional para representaciones teatrales y disponer sillas para ubicar al público e instalación del sonido para poder musicalizar las obras de teatro que serían representadas.

Acción 3: Previo a la representación los jóvenes se prepararon en un 
espacio acondicionado como camerino para vestirse y maquillarse. Allí desarrollaron todo lo aprendido en el Taller de Maquillaje. Este espacio no sólo fue un espacio creativo, sino también de colaboración, de trabajo cooperativo, pues los familiares se unieron en función de apoyar a los jóvenes para desarrollar su experiencia artística.

Llegado el momento de la representación, pudimos contar con la presencia de estudiantes de la Escuela de la comunidad, padres y representantes y demás miembros de la colectividad quienes quedaron muy satisfechos y entusiasmados con la actividad. Las obras se desarrollaron en un tiempo de duración de cincuenta y cinco minutos (55 $\mathrm{min}$ ) y dejaron una gran cantidad de enseñanzas y reflexiones entre el público que estuvo presente. Finalmente se entregaron credenciales a todos los participantes quienes culminaron el proceso con mucho entusiasmo.

\subsection{Reflexiones concluyentes}

La investigación, evidenció que la disciplina teatral, es una herramienta para la integración, comunicación, y participación dentro del ámbito comunitario, capaz de contribuir y aperturar espacios de discusión reflexiva entre jóvenes que se encontraban en estado de vulnerabilidad y que desde el ejercicio creativo y socializador, se abrieron al entendimiento de la importancia de estudiar, leer y escribir inmersos en una experiencia formativa teatral. Fue a partir de allí que la convivencia en la práctica teatral se desenvolvió en un ambiente cordial y de intercambio de opiniones que generó un espacio de franqueza y creatividad que se tradujo en cambios sustanciales para los participantes debido a que, siendo una actividad práctica, donde la puesta en escena los llevó a establecer lazos de amistad y camaradería, se evidenciaron cambios.

En cuanto al proceso de Evaluación de cada una de las Fases, resultó necesario diseñar un instrumento pensando en función de posibilitar en los 
participantes el desarrollo de sus habilidades de reflexión, observación, capacidad de análisis y desarrollo de la capacidad crítica y la resolución de problemas. Nuestro objetivo fue ejecutar un sistema de evaluación que nos permitiera mejorar la propuesta pedagógica y ajustar sobre la marcha lo que fuera necesario para entender el proceso a manera de alcanzar la consecución de las metas o expectativas previstas. De allí que se diseñara un formato del que se expresa un fragmento en la tabla 2, el cual resultara apropiado para la evaluación de las fases permitiendo realizar las transformaciones que resultasen necesarias.

Tabla 2. Fragmento del formato del Plan de Evaluación.

\begin{tabular}{|l|l|}
\hline OBJETIVOS ESPECÍFICOS & $\begin{array}{l}\text { Orientar acerca de las técnicas y formas expresivas que } \\
\text { se utilizan en la escritura dramática. }\end{array}$ \\
\hline FECHA & 16 de septiembre del año 2017. \\
\hline & $\begin{array}{l}\text { - Conversatorio acerca de la importancia de escribir. } \\
\text { - Participación espontánea y sugerida de los jóvenes que } \\
\text { conforman el grupo de trabajo. }\end{array}$ \\
- Breve explicación acerca de las formas de abordar la \\
escritura de un texto teatral. \\
DE LO OBSERVADO & $\begin{array}{l}\text { Al inicio del conversatorio hubo mucha timidez en } \\
\text { participar, pero algunos se expresaron debido a que se } \\
\text { les dio la libertad de hacerlo cuando sintieran la } \\
\text { necesidad. Inicio con preguntas acerca de cómo veían su } \\
\text { comunidad y luego se fue introduciendo el tema de la } \\
\text { importancia de escribir. }\end{array}$ \\
\hline CODIFICACIÓN & $\begin{array}{l}\text { - Timidez. } \\
\text { - Poca participación. } \\
\text { - Expresión de ideas. } \\
\text { - Libre participación. } \\
\text { - Socializar el tema de la escritura. }\end{array}$ \\
\hline INTERPRETACIÓN DE LO & $\begin{array}{l}\text { Los participantes vivenciaron inicialmente la experiencia } \\
\text { con timidez, pero a medida en que iban avanzando en el } \\
\text { desarrollo de la actividad, se fueron generando espacios } \\
\text { de confianza y se abrió la oportunidad de expresar sus } \\
\text { ideas. } \\
\text { Participaron de manera espontánea y llegaron a acuerdos } \\
\text { en relación al tema de la escritura, por lo que cada uno } \\
\text { fue aportando ideas en relación a la experiencia de } \\
\text { escribir una historia en equipo para ser representada. }\end{array}$ \\
\hline
\end{tabular}

Fuente: El Autor (2017). 
El formato permitió apreciar cada una de las actividades desarrolladas en las acciones establecidas con su respectivas fechas y horas de ejecución, donde se describió, no sólo lo que se observaba en el transcurso de las acciones y sus procesos pedagógicos, sino también la interpretación de la experiencia a partir de lo observado, todo ello con el propósito de sistematizar lo vivenciado con miras a mejorar las dinámicas, en la medida en que se tenían mayores apreciaciones del grupo y sus prácticas.

\section{Conclusiones}

La experiencia de haber desarrollado este trabajo de investigación afianzó la importancia del teatro como un medio de expresión y comunicación por excelencia en el que se pueden fortalecer relaciones comunitarias que requieren de integración y cooperativismo. En esta experiencia se evidenció la importancia del teatro educativo como una herramienta eminentemente creativa, pedagógica y recreativa que contribuye en la formación de ciudadanos para el desenvolvimiento en conjunto y en beneficio de un bien común. Ha sido entonces una vivencia recreativa-educativa-comunitaria, porque ha demostrado que sí es posible establecer una vinculación entre miembros de una comunidad al atender una necesidad y ejecutar acciones en beneficio de cambiar una situación problemática.

Resulta fundamental el haber establecido contacto con el Consejo Comunal del "Chama" y junto a ellos haber determinado las circunstancias que permitieron la planificación e implementación de un plan de acción o intervención orientado hacia la aplicación de una experiencia, donde el Teatro, se convirtiera en una herramienta formativa, en piedra angular para dar un primer paso al propósito de generar una vinculación entre los jóvenes adolescentes en estado de vulnerabilidad y su comunidad.

La comunicación en la colectividad del "Chama", en los últimos años, ha venido quebrantada por el desinterés, la desidia y muchas debilidades de 
orden público que, no solo en la ciudad de Mérida, sino en el resto del país, cada día distancian a los vecinos de la realidad que viven sus niños y jóvenes al enfrentar la deserción escolar, al igual que de los propósitos que deberían contribuir a una mayor integración con su entorno. Es este el escenario de los ciudadanos que habitan en la zona del "Chama", quien al dar prioridad a proyectos que les conduzca a solucionar sus problemas de infraestructura, alimentación, salud, como ocurre en cualquier comunidad de estas características en nuestro país, ha dejado a un lado la posibilidad de atender a sus jóvenes, que habían venido desertando del sistema educativo, quedándose desocupados y vulnerables a problemas de alcohol, droga y robo, entre otros, en la zona.

La ejecución del Plan de Acción, demostró que sí es posible establecer una mayor vinculación comunitaria, por lo que se propuso como una alternativa para integrar, para abrir la puerta al trabajo en conjunto, así como también a motivar a los jóvenes a participar en actividades donde puedan reconocerse a sí mismos y descubrir nuevas formas para expresarse desde una experiencia creativa y de comunicación como lo es el teatro, desde donde se puede crear un discurso dirigido a la comunidad en torno a los valores fundamentales que son necesarios para alcanzar la unidad e integración como una opción para generar mayores acercamientos.

El teatro históricamente ha sido un instrumento para la expresión, comunicación y consolidación de intereses comunes, especie de puente para que el colectivo exprese su voz creando relaciones más productivas, en la medida en que se convierte en espejo del contexto al presentar problemas, realidades y también soluciones y llamados a la reflexión con miras a transformar las situaciones problemáticas en un escenario de logros en beneficio de todos. Fue este uno de los mayores propósitos del plan de acción, el que se vivenciara una experiencia recreativa y formativa con intenciones comunitarias como punto de partida de lo que serán otras propuestas desde 
lo educativo, cultural y lo recreativo con firmes propósitos de integración de los ciudadanos en las comunidades

No se había desarrollado una actividad como esta en este contexto y fue desde la propuesta de este trabajo de investigación que se generó la oportunidad de valerse del Teatro Educativo como una herramienta para motivar una experiencia de vinculación, donde comenzó a sembrarse la expectativa por verlo posible, por dar un primer paso que abriera la puerta a nuevos proyectos que faciliten encontrar una mayor unificación entre los miembros de la comunidad del "Chama".

\section{Referencias}

Capitillo, J., Urribarri, A., \& Materano, O. (2014). Aplicabilidad del proyecto educativo integral comunitario para la integración de la escuela, familia y comunidad. Sustentabilidad al día, (4), 1-13, pp. 201202TR4202. Venezuela: Universidad Valle del Momboy.

Cruz, P. (2014). El juego teatral como herramienta para el tratamiento educativo y psicopedagógico de algunas situaciones y necesidades especiales en la infancia. Tesis doctoral. España: UNED. Recuperado de:

http://e-spacio.uned.es:8080/fedora/get/tesisuned:EducacionPcruz/Documento.pdf

Flores, E., Loaiza, A., \& Rojas de Ricardo, G. (2020). Rol del docente investigador desde su práctica social. Revista Scientific, 5(15), 106128, e-ISSN: 2542-2987. Recuperado de:

https://doi.org/10.29394/Scientific.issn.2542-2987.2020.5.15.5.106-128

Padrón, J. (2001). La Estructura de los procesos de investigación. Revista Educación y Ciencias Humanas, IX(17), ISSN: 1315-1762. Venezuela: Universidad Nacional Experimental "Simón Rodríguez"

Palmero, M. (2008). La teoría del aprendizaje significativo en la 
perspectiva de la psicología cognitiva. Primera edición, ISBN: 97884-8063-290-4. Barcelona, España: Ediciones Octaedro, S.L.

Strauss, A., \& Corbin, J. (2002). Bases de la investigación cualitativa: Técnicas y procedimientos para desarrollar la teoría fundamentada. Primera edición, ISBN: 978-958-655-624-8; ISBN: 978958-714-513-7. Colombia: Editorial Universidad de Antioquia; Sage Publications, Inc.

UNICEF (2018). Aprendizaje a través del juego: Reforzar el aprendizaje a través del juego en los programas de educación en la primera infancia. New York, Estados Unidos: Fondo de las Naciones Unidas para la Infancia. Recuperado de:

https://www.unicef.org/sites/default/files/2019-01/UNICEF-Lego-

Foundation-Aprendizaje-a-traves-del-juego.pdf

Vargas, C. (2015). El juego en el aprendizaje. [Sitio Web]. México: Revista Vinculando Recuperado de: http://vinculando.org/educacion/juego-enaprendizaje.html 


\section{Igor Raphael Martínez Rebolledo}

e-mail: igorm@ula.ve

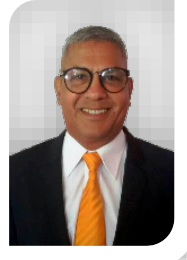

Nacido en Caracas, Venezuela el 25 de mayo del año 1963.

Profesor en Artes Plásticas y Diseño; Licenciado en Pedagogía Alternativa; Especialista en Estudios Teatrales y Cinematográficos; Especialista en Educación para la Gestión Comunitaria; Magister Scientiarum en Ciencias de la Educación; Magister Scientiarum en Gestión para la creación de espacios intelectuales; Doctor en Ciencias de la Educación; Profesor ordinario en categoría de Asistente, en el Departamento de Actuación; Escuela de Artes Escénicas, de la Facultad de Arte en la Universidad de Los Andes (ULA), Mérida, Venezuela; Investigador PEI-ONCTI A2, PEI-ULA.

El contenido de este manuscrito se difunde bajo una Licencia de Creative Commons ReconocimientoNoComercial-Compartirlgual 4.0 Internacional 